\title{
Persistence of the effects of choice on paired-associate learning
}

\author{
RICHARD A. MONTY $\dagger$ \\ Human Engineering Laboratory, Aberdeen Proving Ground, Maryland 21005 \\ and \\ LAWRENCE C. PERLMUTER \\ Virginia Polytechnic Institute and State University, Blacksburg, Virginia 2406 I
}

\begin{abstract}
Four experiments are reported in which half of the subjects were permitted to choose either the stimulus or response members of S-R pairs from groupings of alternative stimuli or responses following the procedures of Perlmuter, Monty, and Kimble. The other subjects were exposed to the materials and were forced to learn the material chosen by their yoked partners. Approximately $24 \mathrm{~h}$ later, both groups learned PA lists comprised of either the chosen or forced material. Choosing either the stimulus or the response facilitated performance irrespective of whether the other member of the S-R pair was present at the time of choosing. This finding ruled out an associative hookup interpretation in favor of a purely motivational hypothesis and demonstrated the temporal durability of the effect of choosing.
\end{abstract}

Previous experiments have defined a number of conditions in which performance can be improved by permitting subjects to choose either the stimulus or the response side of $S-R$ pairs immediately prior to paired-associate (PA) learning (Monty \& Perlmuter, 1972; Monty, Rosenberger, \& Perlmuter, 1973; Perlmuter, Monty, \& Kimble, 1971). Basically, in some of these experiments one group of subjects was permitted to choose from among alternative responses and thereby compose $S \cdot R$ pairs to be subsequently learned in a PA paradigm. Yoked partners viewed the same materials but were forced to learn the chosen S-R pairs, i.e., they had no opportunity to exercise choice. Performance of the subjects who were allowed to exercise choice was generally superior to those denied this opportunity.

A seemingly obvious explanation for the beneficial effect of choice would be that the subjects doing the choosing had an opportunity to form S-R associative hookups which were not necessarily to their yoked partner's liking. There is evidence that the choice phenomenon cannot be explained either by associational or attentional factors. Perhaps the most salient evidence against these alternatives was produced by the study of Monty et al (1973), which demonstrated that, if the point at which choice takes place is properly manipulated, choosing only three responses in a 12 -item list elevates performance to a level near that in which all responses are chosen. In other words, the amount of

\footnotetext{
*This paper may be reproduced in full or in part for any purpose of the United States Government. The assistance of Meg White and Jill Kastris in running the subjects of these experiments is grate fully acknowledged.

+Please address requests for reprints to Richard A. Monty, Behavioral Research Directorate, U.S. Army Human Engineering Laboratory, Aberdeen Proving Ground, Maryland 21005.
}

material chosen is not nearly as important as the point at which choice is exercised. This would seem to argue that the beneficial effects of choice operate to increase motivation and thereby benefit performance. Further evidence for the nonassociative explanation is derivable from Smith and Brown (1968), who showed in a serial learning task that the development of correct anticipations for both preferred and nonpreferred words was enhanced by prior choice. This effect occurred for both high- and low-meaningfulness materials, although it was significant only for the latter. However, one difficulty with the proposed motivational interpretation stems from an experiment by Perlmuter et al (1971), who found that choosing responses in the absence of their respective stimuli did not benefit performance. And interestingly, Perlmuter and Monty (1973) demonstrated that, when subjects chose the stimulus words in the absence of the response words, performance was even poorer than that of subjects given no choice at all. Thus, it appears that in some cases the act of choosing may induce negative aspects into the learning situation as well as positive. More recently, Perlmuter, Monty, and Cross (1974) demonstrated that the beneficial effects of choosing are also destroyed when an intervening list is presented prior to the learning of the chosen list. It is possible, however, that the passage of time was responsible for the equalization of performance on the learning of the chosen list, as the temporal stability of the often observed beneficial effect of choice had not been established.

The major purpose of the present experiments was to determine whether a separation in time between the choice procedure and the commencement of a PA learning trial modifies the previously demonstrated effects of choosing. 

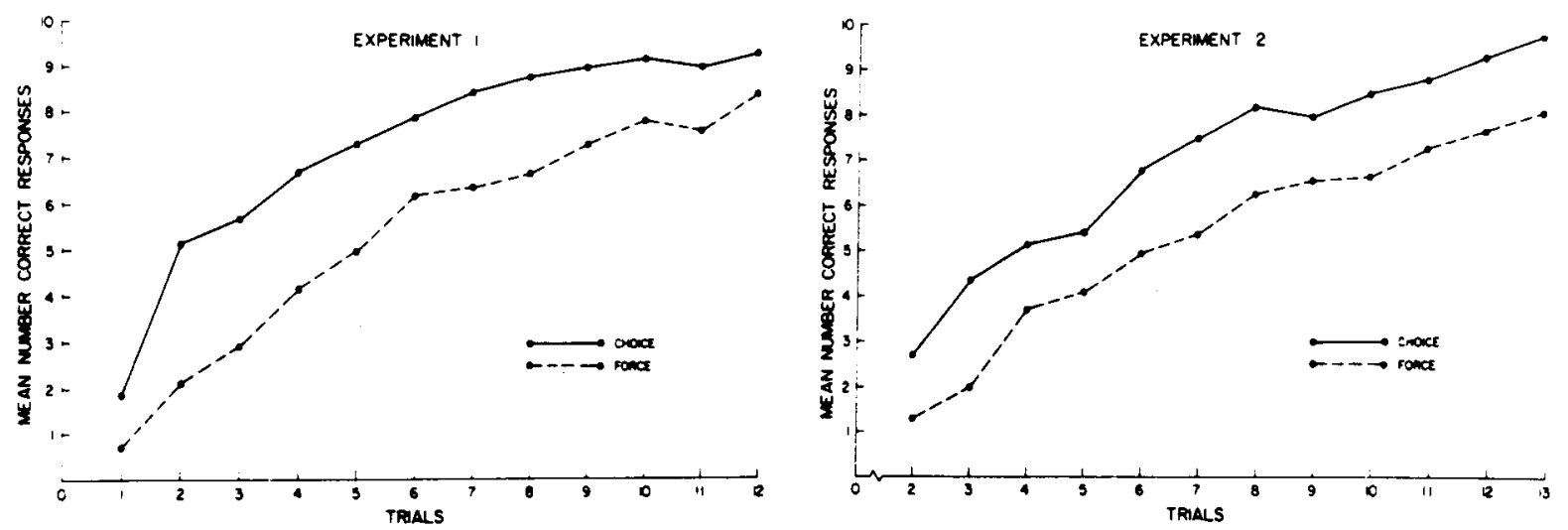

Fig. 1. Mean number of carrect responses as a function of groups and trials for Experiment 1 (choosing stimuli in the presence of responses) and Experiment II (choosing stimuli in the absence of responses).

\section{EXPERIMENT I}

Perlmuter and Monty (1973) have shown that choosing stimuli in the presence of their respective responses immediately prior to PA learning facilitates performance. The purpose of the first experiment was to examine the same phenomenon when choosing takes place a day prior to the learning of the PA list.

\section{Method}

The subjects were 32 male and female volunteers from Virginia Polytechnic Institute and State University summer school who received academic credit for their participation in the experiment.

The stimuli consisted of words typed in elite capitals on Kodak Ektagraphic write-on slides. The slides were presented with a Kodak 760 Carousel projector programmed with a Hunter Model $111 \mathrm{C}$ silenced decade interval timer at a viewing distance of approximately $1.8 \mathrm{~m}$. Sixty five-letter highly meaningful stimulus words were taken from the list generated by Taylor and Kimble (1967). The 60 words were assembled into 10 groups of six items each. Within each group, one word was designated arbitrarily as the response word and the remaining five words served as the five potential stimulus words.

The subjects were divided into two groups of 16 subjects each on the basis of the order of their arrival in the laboratory. Each subject was first presented with three PA trials utilizing common everyday words to be sure they understood the concept of PA learning. The choice group was then shown 10 slides, one at a time. Each slide contained five potential stimulus words listed vertically on the left and a response word centered on the right. The subject was instructed to read aloud the five potential stimulus words and the response word and then to choose one word to serve as the stimulus word to that particular response word. Each of the subjects went through each of the 10 slides at his own rate. The time of presentation per slide was approximately $15 \mathrm{sec}$.

On the following day, subjects were presented with $12 \mathrm{PA}$ learning trials comprised of their individually selected S-R pairs. The stimulus and S-R slides were shown for $2 \mathrm{sec}$ each. The interval between slides was approximately $.9 \mathrm{sec}$. The subject was instructed to anticipate aloud the appropriate response word upon presentation of each stimulus slide. The 10-pair list was presented in three different random orders, and each subject viewed all items in the same order. A brief period of about $2 \mathrm{sec}$ followed every third presentation of the list to enable the experimenter to return the tray to its starting position. The time between the first and second session ranged from about 19 to $29 \mathrm{~h}$, with a mean of about $24 \mathrm{~h}$.

The force group was treated identically to the choice group except that, although they read the stimulus and response words aloud, they were not given the opportunity to choose their own stimuli. Rather, following their reading, they were assigned the stimulus words chosen by the previously tested choice subject while each slide was still visible to them.

To insure that results were not confounded on the PA trials by position effects, each subject was presented with a different random order of the potential S-R pairs during the pre-PA learning procedure. All subjects were informed prior to the start of the experiment that they were required to return on the following day.

\section{Results}

The number of words correct per trial served as the measure of performance. A one between- and one within-variable analysis of variance was performed on the data to determine if there were any differences in performance on the list as a function of groups (choice vs force) or trials. The main effects for groups $[F(1,30)=15.44, p<.001]$ and trials $[F(11,330)=$ $114.06, p<.001]$ were both significant. The data underlying the significant Groups by Trials interaction $[F(11,330)=2.31, p<.025]$ are shown in Fig. 1. It can be seen that the choice group was generally superior to the force group but that the difference diminished slightly with practice or trials

This finding is similar to that reported by Perlmuter and Monty (1973). Thus, it appears that the beneficial effects attributed to having had the opportunity to choose are not vitiated by time, at least when subjects choose the stimulus in the presence of the response. To determine whether retention was affected by choice, an analysis of performance on Trial 1 was made. Here also it was found that the choice group performed reliably better than the force group $[\mathrm{F}(1,30)=5.36, \mathrm{p}<.05]$, demonstrating thereby that choice benefits not only performance but may also benefit retention. 


\section{EXPERIMENT II}

Perlmuter and Monty (1973) also found that choosing the stimuli in the absence of the responses fails to bring about the positive effects previously attributed to choice. In fact, this condition actually resulted in the force group outperforming the choice group, suggesting that motivational factors may induce negative aspects into the learning situation as well as positive ones. The purpose of the second experiment was to determine if these factors also persist with time.

\section{Method \\ The subjects were 32 experimentally naive male and female students from Virginia Polytechnic Institute and State University summer school who received academic credit for their participation in the experiment. \\ The apparatus and procedures were generally identical to those employed in Experiment I, with the following exceptions: Each group of five potential stimulus words was shown to the subjects in the absence of the appropriate response words. Thus, the choice group $(\mathrm{N}=16)$ selected their stimulus words in the absence of the response words and the force group $(\mathrm{N}=16)$ was also exposed to all of the stimuli but was required to learn those stimuli selected by the choice subjects. In addition, whereas the stibjects were unable to anticipate the correct responses on the first PA trial, they were given an additional trial, for a total of 13. The PA trials were presented approximately $24 \mathrm{~h}$ after the choice-force procedure, as in Experiment I.}

\section{Results}

As in the previous experiment, the number of correct responses per trial was subjected to an analysis of variance, with groups as a between effect and trials as a within effect. The data from the first trial were omitted from the analysis, as all scores were zero. The data underlying the analysis are shown in Fig. 1. The main effect for groups $[F(1,30)=7.68, p<.01]$ reached significance, indicating that the choice group was generally superior to the force group, while the main effect for trials $[F(11,330)=100.62, p<.001]$ indicated that performance improved with practice or trials. In contrast to Experiment I, the Groups by Trials interaction failed to reach significance at the .05 level of confidence.

The present data are in sharp contrast to those of Perlmuter and Monty (1973), which showed that, when subjects choose stimuli in the absence of the response and then are required to immediately learn the PA list, force subjects perform better than choice subjects. These results (Perlmuter \& Monty, 1973) could lead one to speculate that, in order for choice to benefit performance, it is necessary that subjects be permitted to choose the S-R pairs, presumably because it gives subjects the opportunity to form S-R associations during the choice procedure. The design of the present experiment prevents this and further adds to the body of literature contesting the associative hookup interpretation in favor of a purely motivational interpretation. Of course. it remains necessary to explain the reason for the diametric opposition between the present data and those reported earlier (Perlmuter \& Monty, 1973). In the earlier study, the subjects chose stimuli in the aisence of the responses during the final part of the experiment and, within a few minutes of this choosing, the respactive responses were assigned. In the present study. approximately $24 \mathrm{~h}$ intervened between the choosing of stimuli and the assigning of responses. This will be discussed further following Experiment IV.

\section{EXPERIMENT III}

Thus far we have investigated only the stimulus side of the S-R pair. There is evidence, however, to suggest that the effect of many variables is not identical on both the stimulus and response sides of the S-R pair (Noble, 1963; Perlmuter \& Monty, 1973; Yarmey \& Sayer, 1972). The purpose of the third experiment was to examine the temporal persistence of the choice effect when responses are chosen in the presence of the stimuli.

\section{Method}

The subjects were 42 experimentally naive male and female volunteers from Virginia Polytechnic Institute and State University summer school who received academic credit for their participation in the experiment.

The apparatus and general procedures were identical to those of Experiment I, except that the choice subjects were given the opportunity to choose one of the five potential response words in the presence of the respective stimuli and the force subjects were required to learn the response words of the choice subjects. A 12-word list was used in this experiment. All subjects returned after approximately $24 \mathrm{~h}$ to begin the PA trials.

\section{Results}

As in the previous experiments, the results were subjected to an analysis of variance, with groups as a between effect and trials as a within effect. The main effects for groups $[F(1,40)=8.14, p<.01]$ and trials $[F(11,440)=207.54, p<.001]$ both reached significance, as did the Group by Trials interaction $[F(11,440)=3.00, p<.01]$. The data are shown in Fig. 2. It can be seen that the choice group consistently performed better than the force group. The magnitude of the superiority of the choice group tended to rapidly increase with trials and subsequently diminish as performance of both groups neared asymptote. Thus, the beneficial effects of choosing responses in the presence of stimuli that were apparent immediately following the act of choosing (Monty et al, 1973; Monty \& Perlmuter, 1972; Perlmuter et al, 1971) also endure a 24 -h interval. Further, as shown by subsequent analysis of Trial $1[F(1,40)=4.13, p<.05]$, retention for chosen responses is superior to that for forced responses.

\section{EXPERIMENT IV}

Experiment II demonstrated that subjects who choose stimuli in the absence of responses show superior PA 

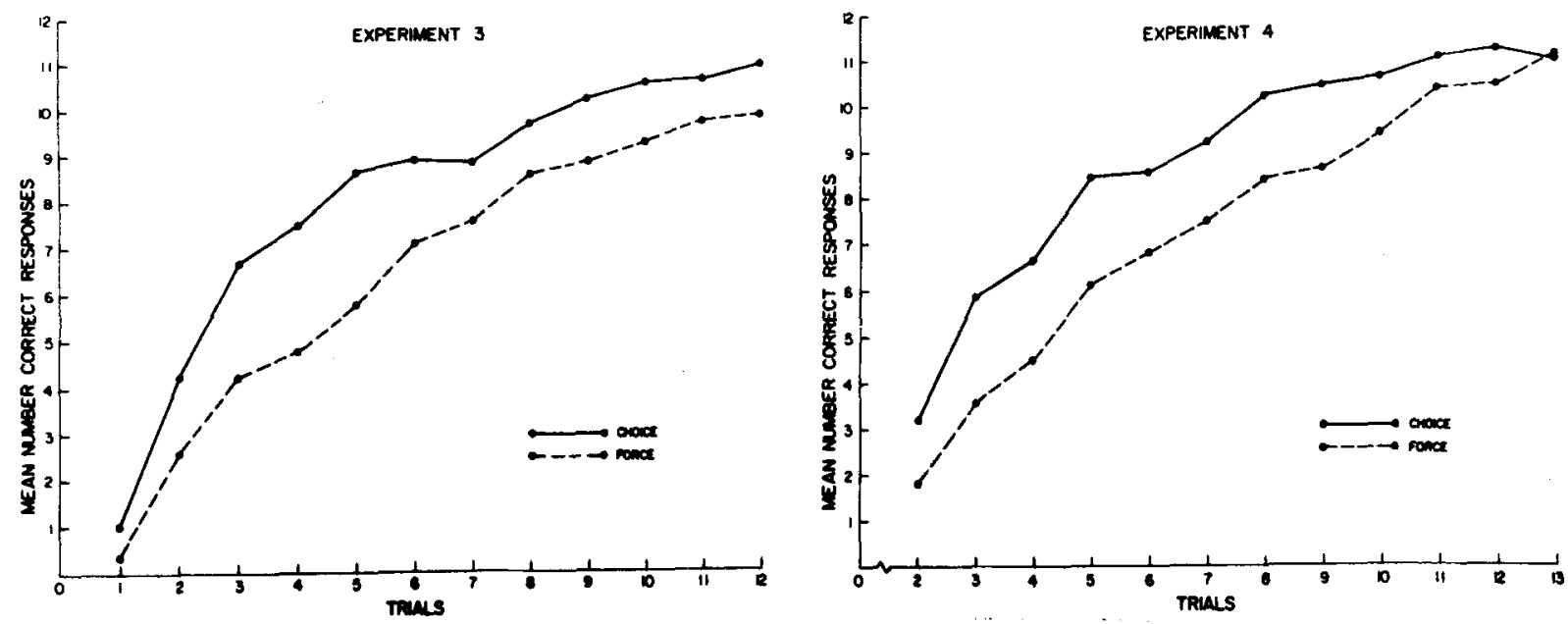

Fiz 2. Mean number of correct responses as a function of groups and trials for Experiment III (choosing responses in the presence of stimuli) and Experiment IV (choosing responses in the absence of stimuli).

performance if a time interval of approximately $24 \mathrm{~h}$ elapses between the act of choosing and the PA learning trials. The purpose of the present experiment was to determine if similar beneficial results occur when subjects choose responses in the absence of stimuli. Perlmuter et al (1971) found no difference between choice and force subjects when PA learning immediately followed the act of choosing.

\section{Method}

The subjects were 38 experimentally naive male and female students from Virginia Polytechnic Institute and State University summer school who received academic credit for their participation in the experiment.

The apparatus and procedures were generally identical to those of Experiment III, except that subjects chose or were assigned their responses in the absence of the respective stimulus items. The subjects returned after approximately $24 \mathrm{~h}$ to begin the PA trials.

\section{Results}

As in the previous experiments, the results were subjected to an analysis of variance, with groups as a between effect and trials as a within effect. The main effects for groups $[F(1,36)=5.06, p<.05]$ and trials $[F(11,396)=150.36, p<.001]$ were both significant, as was the Groups by Trials interaction $[F(11,396)=$ $2.87, \mathrm{p}<.011$. The data are shown in Fig. 2. It can be seen that early in the PA learning trials the choice group was superior to the force group but that the superiority diminished as performance neared asymptote. Once again it appears that beneficial effects of choosing are apparent after a temporal interval of approximately $24 \mathrm{~h}$, although these effects were not apparent after the act of choosing (Perlmuter et al, 1971).

\section{DISCUSSION}

When subjects chose either the stimulus words in the presence of the response words (Perlmuter \& Monty, 1973) or the response words in the presence of the stimulus words (Monty et al, 1973) immediately prior to PA learning, the act of choosing facilitated performance. Experiments I and III serve to extend these findings and have demonstrated that, if an interval of approximately $24 \mathrm{~h}$ elapses between choosing and PA learning, then the beneficial effects of choosing persist. On the basis of a number of experiments (e.g., Monty et al, 1973), it has been suggested that allowing a subject to choose at least a portion of the materials to be learned leads to an increment in the subject's motivational level. The results of Experiments I and III are not incompatible with this interpretation. The results of Experiments II and IV lend additional support to the persistence of the choice effect but, more importantly, also suggest a motivational interpretation of that effect. That is, choosing the stimulus in the absence of the response or the response in the absence of the stimulus disallows the associative interpretation. Hence, on the basis of these and other data, the most parsimonious explanation appears to be a motivational one.

The beneficial effects of choice demonstrated in Experiments II and IV seemingly contradict some of the results reported earlier. Specifically, Perlmuter et al (1971) reported that, when subjects chose the response in the absence of the stimuli, no beneficial effects of choice were apparent. Further, Perlmuter and Monty (1973) demonstrated that, when subjects chose stimuli in the absence of responses, the force subjects actually performed better than the choice subjects. And, finally, when subjects chose responses in the presence of the stimuli prior to learning an A-B list but were forced to 
learn an A-C list either immediately before A-B (Perlmuter et al, 1974) or immediately after A-B (Perlmuter et al, 1971), force subjects performed better than choice subjects. The latter observations were attributed to an additional but nonoptimal increase in motivation of the choice subjects stemming from frustrative effects of the imposition of the nonchosen A.C list.

Senkowski (1973) has presented some evidence that frustration effects monotonically dissipate with time. Although the present experiments do not directly address this problem, it is hypothesized that the potential for frustration may also dissipate over time and that this dissipation may account for the discrepancies between the previously reported studies and Experiments II and IV of the present series. Specifically, when subjects choose stimuli in the absence of the respective responses and immediately are assigned responses, this may serve as a source of frustration to the choice subjects and cause a degradation in their performance due to a nonoptimal increment in motivation. If, however, $24 \mathrm{~h}$ intervene between the choice of the stimuli (in the absence of the respective responses) and the assignment of responses, the potential for frustration will have dissipated, allowing for the expression of the durable effects of choice. However, choice of the response in the absence of the stimulus immediately preceding the A-B learning trials does not disrupt performance of the choice subjects and, hence, does not confirm this explanation. Perhaps the assignment of the less active (stimulus) member of the $S-R$ pair is less disruptive to the subject. In any case, this discrepancy will require further study. Prior evidence has been presented showing that the effects of some variables are not identical on the stimulus and response sides of the S-R pair (e.g., Yarmey \& Sayer, 1972).

In conclusion, the data are important for these reasons: First, the observation by Perlmuter et al (1974) that the beneficial effects of choosing are destroyed when an intervening list is presented prior to the learning of the chosen list cannot be accounted for by the passage of time in that we have seen that the effects persist over an interval of $24 \mathrm{~h}$. Second, the observation that beneficial effects are apparent even when the stimuli or responses are chosen in the absence of their respective S-R unit indicates that, in contrast to previous interpretations (Perlmuter \& Monty, 1973; Perlmuter et al, 1971), the act of choosing may be all that is required for the operation of the suggested motivational mechanism. In other words, the beneficial effects of choice are apparent even when subjects have no opportunity to form associative hookups during the act of choosing. Third, the remarkable persistence of the beneficial effects of choice suggests that the phenomenon may have some practical implications for the field of education. Finally, there is an important parallel developing between the work reported here and that of Glass and Singer (1972). These investigators have reported that exposure to a variety of aversive stimuli such as noise or electric shock resulted in detrimental behavioral aftereffects. They conclude, however, that it is not the physical parameters of the noxious stimuli which bring about performance decrements, but rather the frustration effects from the subject's perception of lack of control over his environment. Lefcourt (1973) has recently reviewed a large body of literature and comes to essentially the same conclusion-that perceived control is the important ingredient. Our own research would seem to generalize the importance of control even further in that the force condition used in our paradigm would probably not be considered noxious in the sense of Lefcourt's review. In Lefcourt's words, "The sense of control, the illusion that one can exercise personal choice, has a definite positive role in sustaining life [p. 424]."

\section{REFERENCES}

Glass, D. C., \& Singer, J. E. Behavioral aftereffects of unpredictable and uncontrollable aversive events. American Scientist, 1972, 60, 457-465.

Lefcourt, H. M. The function of the illusions of control and freedom. American Psychologist, 1973, 28, 417-425.

Monty, R. A.. \& Perlmuter, L. C. The role of choice in learning as a function of meaning and between- and within-subjects designs. Journal of Experimental Psychology, 1972, 94, 235-238.

Monty, R. A., Rosenberger, M. A., \& Perlmuter, L. C. Amount of locus of choice as sources of motivation in paired-associate learning. Journal of Experimental Psychology, 1973, 97, 16-2l.

Noble, C. E. Meaningfulness and familiarity. In C. N. Cofer and B. S. Musgrave (Eds.), Verbal behavior and learning: Problems and processes. New York: McGraw-Hil, 1963.

Perlmuter, L. C., \& Monty, R. A. Effect of choice of stimulus on paired-associate learning. Journal of Experimental Psychology, $1973,99,120-123$.

Perlmuter, L. C., Monty, R. A., \& Cross, P. M. Choice as a disrupter of performance in paired-associate learning. Journal of Experimental Psychology, 1974, 102, 170-172.

Perlmuter, L. C., Monty, R. A., \& Kimble, G. A. Effect of choice on paired-associate learning. Journal of Experimental Psychology, 1971, 91, 47-53

Senkowski, P. C. Temporal aftereffects of reward and nonreward in an operant analogue of the double-alley nunway. Journal of Experimental Psychology, 1973, 101, 348-359.

Smith, R. K., \& Brown, J. Serial acquisition as a function of the meaningfulness (m) of words chosen vs. mean $m$ value of words learned. Psychological Record, 1968, 18, 623-627.

Taylor, J. D., \& Kimble, G. A. The association value of 320 selected words and paralogs. Journal of Verbal Learning \& Verbal Behavior, 1967,6,744-752.

Yarmey, A. D., \& Sayer, L. A. Associative learning of abstract and concrete nouns and their subject-drawn pictorial representation. Canadian Journal of Psychology, 1972, 26, 240-251.

(Received for publication July 21, 1974; accepted July 30, 1974.) 